\title{
Benefits of short duration diagnostic laparoscopy prior to laparotomy in upper gastrointestinal malignancy
}

\author{
Hatem Soltan, MD; Mahmoud Bahram, MD
}

Department of General Surgery, Menofiya University, Egypt.

\begin{abstract}
Introduction: Many patients with upper gastrointestinal malignancies present with locally advanced or metastatic disease and therefore accurate staging assists in the appropriate treatment selection for cure or palliation. Even after modern preoperative imaging studies (abdominal ultrasound, CT scan and MRI), many patients are found to have unsuspected metastases and lor irresectable disease at exploration.

Aim of the work: To evaluate the role of diagnostic laparoscopy in detection of small metastases, and assessment of resectability in patients with upper gastrointestinal malignancies.

Patients and methods: This is a prospective study of 57 patients with primary upper gastrointestinal malignancy, admitted to Menoufyia University Hospital between April 2005 and April 2009. Diagnostic laparoscopy was performed under general anesthesia immediately before scheduled exploratory laparotomy for all patients. The sensitivity and diagnostic accuracy of laparoscopy for distant metastases and tumor resectability were assessed against the findings on preoperative investigations and open surgery.

Results: The mean operative time for diagnostic laparoscopy was $18 \pm 2.387 \mathrm{~min}$. Diagnostic laparoscopy detected metastatic disease in 15/57 (26.3\%) patients, so those patients avoided unneeded exploratory laparotomy. The mean hospital stay for patients in whom diagnostic laparoscopy was done without laparotomy, as patients had metastatic disease was $1 \pm 0.231$ day. Diagnostic laparoscopy was sensitive in detecting the un-resectable tumors in (83.3\%) of patients and this was significantly higher than the sensitivity of preoperative investigation alone (61.4\%) $P<0.05$. The overall morbidity of staging laparoscopy was minimal.

Conclusion: Diagnostic laparoscopy is a safe and effective modality in patients with gastrointestinal tumors. It helps in avoiding unnecessary laparotomies in a significant number of patients.
\end{abstract}

Key words: Diagnostic laparoscopy, metastasis, gastrointestinal malignancies.

\section{Introduction:}

The therapy of upper gastrointestinal (GIT) tumors is becoming more and more complex comprising surgical resection, investigations, neo-adjuvant, adjuvant, palliative chemotherapy or supportive care. The basis of optimized therapies is the correct evaluation of tumor spread and exact staging. ${ }^{1}$

Despite the advances of imaging technology (trans-abdominal and endoscopic ultrasound, CT scan, MRI and PET-CT), noninvasive staging modalities are still inaccurate in identifying small volume metastatic disease (peritoneal tumor spread and occult liver metastases). ${ }^{2}$
For those who do not require a palliative procedure, non-therapeutic laparotomy offers little benefit and may be associated with significant morbidity and mortality affecting both the quality of life and duration of their survival. ${ }^{3}$

In order to minimize the number of patients with imaging occult disease that undergo unnecessary laparotomy, laparoscopy was incorporated early in the staging algorithm of cancer patients. Staging laparoscopy has repeatedly been shown to identify small peritoneal or liver implants not seen on preoperative staging. ${ }^{2}$ 


\section{Patients and methods:}

This is a prospective study of 57 patients with primary abdominal malignancy (gastric, esophageal, pancreatic tumors) between April 2005 and April 2009. Inclusion criteria include patients with esophageal, gastric or pancreatic cancer not complicated by obstruction or distant metastases.

Exclusion criteria include patients with complications related to the cancer (obstruction, bleeding or perforation) and patients with distant metastases or medically unfit (those were referred for palliative therapy or supportive care).

The study protocol was approved by the Ethics Committee, Faculty of Medicine, Menofiya University. All patients received detailed information on diagnostic laparoscopy and only those who agreed to the study protocol were finally enrolled in the study. The pretherapeutic imaging staging included conventional radiography of the thorax, abdominal ultrasonography and abdominal CT or MRI. All patients were felt to be resectable and non metastatic based on preoperative imaging techniques prior to undertaking staging laparoscopy.

The procedure for diagnostic laparoscopy was done under general anesthesia and following the establishment of pneumoperitoneum, a thorough evaluation of peritoneal surfaces is performed; the suprahepatic and infra-hepatic spaces, the surface of the bowel, the lesser sac, the root of the transverse mesocolon and small bowel, the ligament of Treitz, the paracolic gutters and pelvis are inspected with frequent bed position changes as necessary. Malignant ascitic fluid if present (hemorrhagic ascitis) was sent for cytology and biopsies of any suspicious lesions obtained.

The patients with M1 or un-resectable because of local tumor extension were referred for palliative therapy, chemotherapy, chemoradiation and/or supportive care. Open laparotomy was immediately performed in the M0 patients and those with resectable or uncertain resectability.

The specificity, sensitivity and diagnostic accuracy of lymph node, peritoneal and hepatic metastases were assessed against the final pathological report on permanent sections.

\section{Results:}

This is a prospective study of 57 ( 23 female and 34 male) with their ages ranged from 25 to 72 years and the mean age was $57 \pm 14.653$. The primary sites of malignancy were; cancer lower third esophagus in 15 patients, cancer stomach in 18 patients and cancer pancreas in 24 patients. Imaging studies of all patients revealed that all patients were resectable with no lymph node detected or metastatic diseases (liver, peritoneal and lung).

The mean operative time for diagnostic laparoscopy with or without biopsy was $18 \pm 2.387 \mathrm{~min}$. The mean hospital stay for patients in whom diagnostic laparoscopy was done without laparotomy (patients had metastatic disease) was $1 \pm 0.231$ day, Table(1).

Table (1): Operative time and hospital stay for diagnostic laparoscopy patients.

\begin{tabular}{|l|c|c|c|}
\hline & Minimum & Maximum & Mean $\pm \mathrm{SD}$ \\
\hline Operative time & $10 \mathrm{~min}$ & $30 \mathrm{~min}$ & $18 \pm 2.387 \mathrm{~min}$ \\
\hline Hospital stay & 1 day & 2 day & $1 \pm 0.231$ day \\
\hline
\end{tabular}


Table (2): Results of diagnostic laparoscopy.

\begin{tabular}{|c|c|c|c|c|c|}
\hline & $\begin{array}{c}\text { Hepatic } \\
\text { lesion }\end{array}$ & $\begin{array}{c}\text { Peritoneal } \\
\text { nodules }\end{array}$ & $\begin{array}{c}\text { Malignant } \\
\text { ascites }\end{array}$ & $\begin{array}{c}\text { Locally } \\
\text { non- resectable }\end{array}$ & $\begin{array}{c}\text { No. of } \\
\text { avoided } \\
\text { laparotomy }\end{array}$ \\
\hline Lower $1 / 3$ esophagus & $2 / 15$ & $1 / 15$ & 0 & $1 / 15$ & $4 / 15$ \\
\hline Stomach & $1 / 18$ & $4 / 18$ & $1 / 18$ & $1 / 18$ & $7 / 18$ \\
\hline Pancreas & $3 / 24$ & 0 & 0 & $1 / 24$ & $4 / 24$ \\
\hline
\end{tabular}

Table (3): Finding on open laparotomy after diagnostic laparoscopy.

\begin{tabular}{|c|c|c|c|}
\hline & $\begin{array}{c}\text { Resectable on } \\
\text { diagnostic laparoscopy }\end{array}$ & $\begin{array}{c}\text { Resection after } \\
\text { open laparotomy }\end{array}$ & $\begin{array}{c}\text { Sensitivity of } \\
\text { diagnostic laparoscopy }\end{array}$ \\
\hline Lower 1/3 esophagus & 11 & 11 & $100 \%$ \\
\hline Stomach & 11 & 10 & $91 \%$ \\
\hline Pancreas & 20 & 14 & $70 \%$ \\
\hline
\end{tabular}

In this study, a total of 22/57 (36.6\%) patients with upper GIT malignancies were found to be inoperable and or irresectable. Diagnostic laparoscopy before exploratory laparotomy detected metastatic disease in $15 / 57$ (26.3\%) patients so those patients avoided unneeded exploratory laparotomy and its potential complications. Open exploration added another $7 / 57$ (12.3\%) patients with irresectable tumors.

The sensitivity of diagnostic laparoscopy in detecting un-resectable tumors reached $83.3 \%$, as only 7 out of 42 patients had false negative results that lead to unnecessary laparotomy. While the sensitivity of preoperative investigation reached $61.4 \%$ as 22 out of 57 patients had false negative results that leaded to surgical intervention. And this difference was statistically significant $\mathrm{P}<0.05$.

The diagnostic laparoscopy before exploratory laparotomy did not increase the operative time (mean increases $\pm \mathrm{SD}$ was $18 \pm 2.387 \mathrm{~min}$ ) in patients who had no metastatic disease and resection was done but it decreases the hospital stay (mean hospital stay was $1 \pm 0.231$ day) in patients who had metastatic disease and laparotomy was not done.

\section{Discussion:}

Although advances in CT have improved the ability to predict resectability, limitations remain including the sensitivity of CT to detect tumors less than $1 \mathrm{~cm}$ in diameter, thus limiting the detection of peritoneal metastatic deposits, small liver metastases, and peritoneal micrometastases. 4

There is good evidence that videolaparoscopic staging is valuable in certain gastrointestinal (gastric, esophageal and pancreatic) and intra-abdominal lymphomas. The evidence available is all retrospective, but 
of sufficient consistency to indicate that laparoscopic staging adds to the primary (imaging) staging and often alters the clinical stage of the disease and hence the management of the individual patient. 5

In this study diagnostic laparoscopy before exploratory laparotomy detected metastatic disease in 4/24 (16.7\%) patients with non obstructed pancreatic cancer thus avoided unneeded exploratory laparotomy and its potential complications. And this agrees with Jiminez et al, ${ }^{6}$ in their study laparoscopy diagnosed unsuspected metastases in $31.2 \%$ of patients with pancreas cancer, thus avoiding non-therapeutic laparotomy. Other studies indicate that staging laparoscopy can detect un-resectable disease in $20 \%$ to $48 \%$ of patients felt to be resectable by CT scan. ${ }^{7-10}$

Because of the natural progression of gastric cancer, the risk of finding peritoneal implants (M1 disease) at the time of laparotomy is 25$37 \%$ after an otherwise, unremarkable CT scan. Considering the fact that few patients with M1 disease actually develop surgical bleeding or significant gastric outlet obstruction prior to death, a strong argument can be made for laparoscopy in all patients with advanced gastric cancer. 11

In this study diagnostic laparoscopy revealed unsuspected peritoneal lesions in 7 out of 18 patients $(38.9 \%)$ with gastric cancer and this agrees with the study of Muntean et al. ${ }^{1}$ in which an unnecessary laparotomy was avoided in 17 out of the 45 gastric cancer patients with staging laparoscopy (37.8\%).

A controversy exists in the literature about the extent of staging laparoscopy between advocates of a short duration procedure that is based only on inspection of surfaces, ${ }^{6-12}$ and those who believe that a more extensive procedure that includes opening of the lesser sac and assessment of the vessels is in order. ${ }^{13-15}$

In agreement with Luque et al, 12 a short time procedure was done in this study. The advantages of a limited examination include that it can be performed quickly (usually within 10-20 min), can be done through one port, does not require significant expertise, minimizes the risk of potential complications by dissection near vascular structures and has good diagnostic accuracy.
But this disagrees with Stefanidis et al. ${ }^{2}$ who suggest that extended technique may detect vascular invasion and deep hepatic metastasis, which are often missed by visual inspection alone, and thus improve the diagnostic accuracy of staging laparoscopy. This may explain why sensitivity of diagnostic laparoscopy to detect irresectable tumors in this study was the least (70\%) in pancreatic cancer than other tumors, in which extended laparoscopy may be needed to discover vascular invasion.

The main risks of staging laparoscopy are complications related to the surgery and anesthesia, the false negative results that lead to unnecessary laparotomies and the potential adverse oncologic effects. ${ }^{16}$

Although in this study short time laparoscopy was done, the sensitivity of diagnostic laparoscopy in detecting unresectable tumors reached $83.3 \%$, and was significantly higher than the sensitivity of preoperative investigation which reached $61.4 \%$.

\section{Conclusion:}

Diagnostic laparoscopy is a safe and effective staging modality in patients with gastrointestinal tumors. It helps in avoiding unnecessary laparotomies in a significant number of patients.

\section{References:}

1- Muntean V, Mihailov A, Iancu C, Toganel R, Fabian O, Domsa I, Muntean MV: Staging laparoscopy in gastric cancer: Accuracy and impact on therapy. $J$ Gastrointestin Liver Dis 2009; 18(2): 189195.

2- Stefanidis D, Grove KD, Schwesinger WH, Thomas Jr.: The current role of staging laparoscopy for adenocarcinoma of the pancreas: A review. Oxford Journals: Annals of Oncology 2005; 17(2): 189-199.

3- McKinlay R, Sanfiel F, Roth JS: The current role of laparoscopy in staging upper gastrointestinal malignancies. Curr Surg 2005; 62(1): 35-37.

4- Diego C, Reichenbach D, Duerr GD, Venema TL, Sweeney JF, Fisher WE: Value of laparoscopy in the staging of 
pancreatic cancer. J Pancreas (Online) 2005; 6(6): 552-561.

5- Cuschieri A: Role of video-laparoscopy in the staging of intra-abdominal lymphomas and gastrointestinal cancer. Seminars in Surgical Oncology 2001; 20: 167-172.

6- Jiminez RE, Warshaw AL, Rattner DW, et al: Impact of laparoscopic staging in the treatment of pancreatic cancer. Arch Surg 2000; 135: 409-415.

7- Reddy KR, Levi J, Livingstone A, et al: Experience with staging laparoscopy in pancreatic malignancy. Gastrointest Endosc 1999; 49: 498-503.

8- Callery MP, Strasberg SM, Doherty GM, Soper NJ, Norton JA: Staging laparoscopy with laparoscopic ultrasonography: Optimizing respectability in hepatobiliary and pancreatic malignancy. J Am Coll Surg 1997; 185: 34-41.

9- Vollmer CM, Drebin JA, Middleton WD, et al: Utility of staging laparoscopy in subsets of peripancreatic and biliary malignancies. Ann Surg 2002; 235: 1-7.

10-Schachter PP, Avni Y, Shimonov M, Gvirtz G, Rosen A, Czeriak A: The impact of laparoscopy and laparoscopic ultrasonography on the management of pancreatic cancer. Arch Surg 2000; 135: 1303-1307.
11-Romeo Giuli: Staging laparoscopy in gastric cancer. J Surg Oncology 2001; ISSN 19521063.

12-Luque-de Leon E, Tsiotos GG, Balsiger B, Barnwell J, Burgart LJ, Sarr MG: Staging laparoscopy for pancreatic cancer should be used to select the best means of palliation and not only to maximize the resectability rate. J Gastrointest Surg 1999; 3: 111-117.

13-Hunerbein M, Rau B, Hohenberger P, Schlag PM: Value of laparoscopic ultrasound for staging of gastrointestinal tumors. Chirurg 2001; 72: 914-919.

14-Schachter PP, Avni Y, Shimonov M, Gvirtz G, Rosen A, Czerniak A: The impact of laparoscopy and laparoscopic ultrasonography on the management of pancreatic cancer. Arch Surg 2000; 135: 1303- 1307.

15-Rau B, Hunerbein M: Diagnostic laparoscopy: Indications and benefits. Langenbecks Arch Surg 2005; 390: 187 196.

16-Hori Y: Guidelines for diagnostic laparoscopy. Society of American Gastrointestinal and Endoscopic Surgeons (SAGES). Surg Endosc 2008; 22(5):13531383. 